\title{
Models and Strategies on Reopening Lockdown Societies due to COVID-19
}

\author{
Binheng Song ${ }^{1}$, Xiali $\mathrm{Hei}^{2 *}$
}

April 23, 2020

1. Tsinghua University 2. University of Louisiana at Lafayette $*$ corresponding author(s): Binheng Song (songbinheng@ @z.tsinghua.edu.cn) and Xiali Hei (xiali.hei@ louisiana.edu)

\begin{abstract}
COVID-19 has caused community lockdown, factory pausing, and economic activity decreasing. We study a compartmental Susceptible-Infected-Quarantine-Susceptible (SIQS) model, which is derived from the Susceptible-Infected-Susceptible (SIS) model with an infected subject isolation compartment, and get three results through analysis the partial data related to COVID-19. First, we present scientific principles of controlling the spread of COVID-19 via isolation infected subjects. Second, we find that without the lockdown, it is possible to control COVID-19 through isolation more than $15.3 \%$ of unquarantined infected subjects every day. The lockdown communities could be reopened. Third, we propose the strategies to test, isolate the infected subjects under different situations. Some of these strategies could be used to cope with other new pandemic diseases for the human being.
\end{abstract}

\section{Introduction}

In the world, a total of 1,413,415 (source: Johns Hopkins University) COVID-19 cases had been reported from December 1st, 2019 [1] to April 7th, 2020. COVID-19 caused the lockdown of numerous cities and substantial economic losses in many countries. The most effective approaches to mitigate the spread of COVID-19 is lockdown, screening, and isolation. We propose a feasible, large-scale test and isolate the unquarantined infected subject-based control strategies for COVID-19 without affecting the normal life of uninfected subjects. This approach works even under the assumption that there are no available vaccines and long-lasting immunity. With our plan in place, it is possible to mitigate even root out the COVID-19 in society.

\section{The Mathematical Model and Analysis}

The compartmental models in epidemiology are famous and wide-applied in the studies of epidemic diseases. There are many studies on this kind of problems since the Kermack and 
Mckendrick's paper in 1927 [3]. Among them, the Susceptible-Infected-Recovered (SIR) model [4] and Susceptible-Infected-Susceptible (SIS) model [5] are two of the simplest compartmental models, and many models are derivatives of them [6, 7]. The SIS model consists of two compartments: $\mathrm{S}$ for the number of susceptible and $\mathrm{I}$ for the number of infectious. We use $S(t)$ and $I(t)$ to represent the number of susceptible subjects and the number of infectious subjects at the time $t$. We assume the $N=S+I$ is a constant for the SIS model, which is the number of the population in one region. In this paper, we also ignore the number of death because it is insignificant compared to $N$ at the very beginning of the spread of COVID-19.

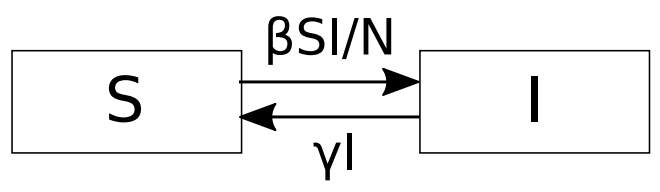

Figure 1: The SIS model

In Fig. 1, the arrows are labeled with the transition rates between compartments. From $\mathrm{S}$ to $\mathrm{I}$, the transition rate (right arrow) is $\beta S I / N$.From I to $\mathrm{S}$, the transition rate (left arrow) is $\gamma I$. $\beta$ and $\gamma$ are constants describing the transition strength between $\mathrm{S}$ and $\mathrm{I}$.

The SIS model without long-lasting immunity described above can be expressed by the following set of ordinary differential equations:

$$
\begin{aligned}
& \frac{d S}{d t}=-\beta S I / N+\gamma I \\
& \frac{d I}{d t}=\beta S I / N-\gamma I
\end{aligned}
$$

where $t$ is time, the unit is one day; the unit of $\beta$ and $\gamma$ is $1 /$ day.

At the beginning of the epidemic, $I \ll N$, thus $S / N \simeq 1$, $\frac{d I}{d t} \simeq(\beta-\gamma) I$. That means $I(t) \simeq I(0) e^{(\beta-\gamma) t}$, and $e^{\beta-\gamma} \simeq \frac{I(t+1)}{I(t)}$ is the ratio of the number of infected subjects on the $(t+1)$ th day to the $t$ th day.

When $\beta>\gamma$, the disease will be pandemic. At the beginning of the pandemic, the number of infected subjects will increase by $100\left(e^{\beta-\gamma}-1\right) \%$, and $\lim _{t \rightarrow \infty} I(t)=\left(1-\frac{\gamma}{\beta}\right) N$.

When $\beta \leq \gamma$, the disease will be extinguished, and $\lim _{t \rightarrow \infty} I(t)=0$.

After adding a compartment of quarantined infected subjects in the SIS model, we get a Susceptible-Infected-Quarantine-Susceptible (SIQS) model. Assume $Q$ is the number of quarantined subjects, which will not contact with susceptible subjects and transmit the virus. In this model, we assume the $N=S+I+Q$ is a constant.

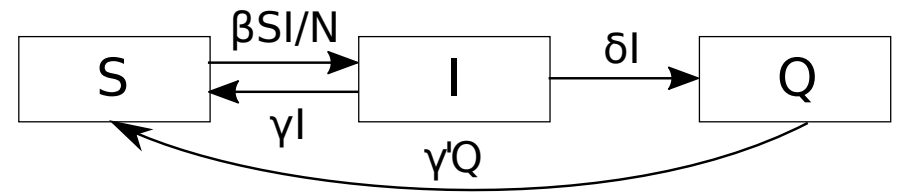

Figure 2: The SIQS Model

Compared with Fig. 1, in Fig. 2, there is one added compartment called the isolation compartment. The number of isolation compartment is $Q$. Also, we assume the transfer 
rate from $I$ to $Q$ is $\delta I$, and the transfer rate from $Q$ to $S$ is $\gamma^{\prime} I$, the corresponding set of differential equations is:

$$
\begin{aligned}
& \frac{d S}{d t}=-\beta S I / N+\gamma I+\gamma Q \\
& \frac{I I}{d t}=\beta S I / N-\gamma I-\delta I \\
& \frac{d Q}{d t}=\delta I-\gamma^{\prime} Q
\end{aligned}
$$

where $\delta$ is the isolation strength of infected subjects at large, $\gamma^{\prime}$ is the recover strength of quarantined subjects. After analysis, we find that:

Theorem 1 : If $\delta>\beta-\gamma$, then $I(t) \leq I(0) e^{(\beta-\gamma-\delta) t}$, that means that if the isolation strength is strong enough, then the number of infected subjects will drop exponentially.

\section{COVID-19 Parameter Estimation}

There are 59,431 confirmed and approximate cases from December 1st, 2019 to February 7th (two weeks after January 23rd, 2020, which is Wuhan lockdown day) in China, and the free transmission time are 67 days. According to above equations, $e^{\beta-\gamma} \simeq 1.178, \beta-\gamma \simeq$ 0.164 for China. Also, there are 392,285 confirmed cases from January 19th, 2019 to April 7th (two weeks after March 23rd, 2020, which is NY pausing day), in the USA, and the free transmission lasts 78 days. According to above equations, $e^{\beta-\gamma} \simeq 1.180, \beta-\gamma \simeq 0.166$ for USA.

Parameter Error Analysis : Since $2^{1 / 78}=1.0089$ and $2^{1 / 67}=1.0104$, when the number of infected subjects being doubled or halved, errors of $e^{\beta-\gamma}$ and $\beta-\gamma$ are around $1 \%$. Note that we use accumulated cases instead of backlog of cases to compute parameters because the negligence of their differences in such situations.

Through our above analysis and estimation, the number of infected subjects increased by $17.8 \%$ and $18.0 \%$ daily in China and the USA, respectively. Since there are other input sources of coronavirus cases in the USA, the actual daily increasing rate will be a little lower than $18.0 \%$. For simplicity, we assume the $e^{\beta-\gamma}=1.180, \beta-\gamma=0.166$ for COVID19 , and we use the number as the base of our control strategies. Actually, different regions may have their own parameters.

Additionally, there are 1,413,415 confirmed cases from December 1st, 2019 to April 7th, 2020, worldwide, and the total free transmission time is 127 days. According to this data, $e^{\beta-\gamma} \simeq 1.119$, which is less than 1.180. One reason behind it is that there are no sufficient tests in many countries. The second reason is that the intervention, especially the national lockdown in China, reduces the daily increasing rate dramatically. 


\section{The Isolation Percentage of Unquarantined Infected Subjects to Control COVID-19}

According to Theorem 1 and the results in Section 3, we know $e^{\beta-\gamma}=1.180$, then, when $\delta>\beta-\gamma=0.166$, we can get $1-e^{-\delta}>1-1 / 1.180=0.153$. That means, if we can find and isolate $15.3 \%$ or more of unquarantined infected subjects every day, we can decrease the number of un-isolated infected subjects exponentially. The larger the $\delta$, the more rapidly the number decreases. Thus, the time needed to control COVID-19 will be shortened rapidly. Table 1 shows the isolation percentage of unquarantined infected subjects under different periods. For example, if we can screen and isolate more than $68.6 \%$ of free infected subjects every week, we can control COVID-19 as well.

\begin{tabular}{|c|c|c|c|c|c|c|c|c|c|c|}
\hline Period T(day) & 1 & 2 & 3 & 4 & 5 & 6 & 7 & 10 & 14 & 20 \\
\hline $100\left(1-e^{-s T}\right)$ & 15.3 & 28.2 & 39.1 & 48.4 & 56.3 & 63.0 & 68.6 & 80.9 & 90.1 & 96.3 \\
\hline
\end{tabular}

Table 1: The isolation percentage under different periods

\section{Suggested Strategy Analysis: Typical Symptom Check and Nucleic Acid Detection}

Assume the possibility of those infected subjects showing symptoms is $a$, the percentage of the population is $b$, the false positive rate of the Nucleic Acid Detection (Fluorescence RT-PCR) is $r$, the false negative rate of the Nucleic Acid Detection (Fluorescence RT-PCR) is $s$, and we can get nucleic acid detection results in real-time.

- Step 1: Conduct COVID-19 symptom screening or self-screening for every unquarantined subjects.

- Step 2: Conduct the nucleic acid detection for subjects with symptoms and isolate subjects with positive results.

If $a(1-s) \geq 16 \%$, COVID-19 will be under control according to results in Section 4; otherwise, this approach will fail. Under this situation, the number of quarantined subjects with false positive results is $b * r$ of the whole population. For example, if the possibility of infected subjects showing a fever is 50\% (lower than 73\% in [2]), $r=5 \%, s=5 \%$ for nucleic acid detections or fast early-tests, and subjects with a fever account for $1 \%$ of the whole population, we can control the spread of COVID-19 when we isolate $0.05 \%$ uninfected subjects and $47.5 \%$ of unquarantined infected subjects.

Feasibility analysis : It is possible to check everyone's body temperature - any individual and institution could run a real-time rapid body temperature check. Besides, the rapid PCR test capability could be increased in months. 


\section{Stategies: How to Effectively Find $15.3 \%$ or More of Unquar- antined Infected Subjects Daily}

Strategy 1: Contact Tracing Since contract tracing cannot find all contacted subjects, it is impossible to prevent the outbreak of COVID-19. However, contact tracing could be combined with other strategies. And we need more contact tracing related work to know the ratio of infected subjects for COVID-19.

Strategy 2: Periodical Nucleic Acid Detection This is the most direct control measure. If we can find more than $15.3 \%$ of unquarantined infected subjects through nucleic acid detection daily, we can control the spread of COVID-19. This approach needs a billiongrade COVID-19 test capability and convenient, accurate COVID-19 tests. This approach is costly; however, the cost is less than economic losses and secondary disasters caused by lockdown in the world. If corresponding mechanisms could be developed over the world, then the human being can handle new or unknown viruses in the future.

Strategy 3: Quarantine the Subjects with Typical Symptoms If all the COVID-19 infected subjects have typical symptoms, we can use this approach to control COVID-19. Otherwise, it will not work when there are many silent carriers.

Strategy 4: Quarantine Confirmed Cases after Test Subjects with Typical Symptoms This approach could be put in place than other strategies. See Section 5.

Strategy 5: Combine Strategy 1 and 4 With the help of contract tracing, we could more efficiently find $15.3 \%$ or more of unquarantined infected subjects and control COVID-19 soon. There are also works on the combined strategy like social distancing and quarantine [8].

\section{Limitation and Future Work}

Based on our SIQS model, we found that COVID-19 could be controlled if we can effectively quarantine $15.3 \%$ or more of unquarantined infected subjects. We also discussed possible approaches to implement them and the feasibility of each strategy.

One limitation is that we have not investigated how to control silent carriers due to lack of data. Additionally, the parameters we used counted on data integrity. The other factors, like hygiene habits, will not affect the conclusion. Note that countries like China have controlled free transmission cases and could reopen the cities by using strategies while strictly screening the input sources. The countries under the breakout of COVID-19 could adopt various procedures until they controlled free transmission cases. Different countries could reopen the border when the input sources are controllable. 
We will work on 1) the effect of input sources, 2) problems considering infected subjects without typical symptoms, 3) the optimization of strategies in the future.

\section{References Cited}

[1] Huang, C., Wang, Y., Li, X., Ren, L., Zhao, J., Hu, Y., Zhang, L., Fan, G., Xu, J., Gu, X. and Cheng, Z., 2020. Clinical features of patients infected with 2019 novel coronavirus in Wuhan, China. The Lancet, 395(10223), pp.497-506.

[2] Bialek, S., Gierke, R., Hughes, M., McNamara, L. A., Pilishvili, T., \& Skoff, T. (2020). Coronavirus Disease 2019 in ChildrenłUnited States, February 12CApril 2, 2020.

[3] Kermack, W.O. and McKendrick, A.G., 1927. A contribution to the mathematical theory of epidemics. Proceedings of the royal society of london. Series A, Containing papers of a mathematical and physical character, 115(772), pp.700-721.

[4] Harko, T., Lobo, F.S. and Mak, M.K., 2014. Exact analytical solutions of the Susceptible-Infected-Recovered (SIR) epidemic model and of the SIR model with equal death and birth rates. Applied Mathematics and Computation, 236, pp.184-194.

[5] Nakamura, G.M. and Martinez, A.S., 2019. Hamiltonian dynamics of the SIS epidemic model with stochastic fluctuations. Scientific reports, 9(1), pp.1-9.

[6] Pongsumpun, P. and Tang, I., 2014. Dynamics of a new strain of the H1N1 influenza a virus incorporating the effects of repetitive contacts. Computational and mathematical methods in medicine, 2014.

[7] Sinha, D.N., 2018. Mathematical Model of Zoonotic Influenza Subtype A (H7N9) Spread in Human Population. J Immunol Tech Infect Dis 7, 1, p.2.

[8] Chowdhury, A., Kabir, K.A. and Tanimoto, J., 2020. How quarantine and social distancing policy can suppress the outbreak of novel coronavirus in developing or under poverty level countries: a mathematical and statistical analysis. 